\title{
Differential effects of low-dose resveratrol on adiposity and hepatic steatosis in diet-induced obese mice
}

\author{
Su-Jung Cho ${ }^{1,2} \dagger$, Un Ju Jung ${ }^{1,2} \dagger$ and Myung-Sook Choi ${ }^{1,2_{*}}$ \\ ${ }^{1}$ Department of Food Science and Nutrition, Kyungpook National University, 1370 Sankyuk Dong Puk-ku, $702-701$ Daegu, \\ Republic of Korea \\ ${ }^{2}$ Center for Food and Nutritional Genomics Research, Kyungpook National University, Daegu, Republic of Korea \\ (Submitted 13 June 2011 - Final revision received 2 November 2011 - Accepted 16 January 2012 - First published online 14 March 2012)
}

\begin{abstract}
Consumption of a high-fat diet (HFD) enriched in saturated fat induces excessive weight gain due to adiposity, which can lead to metabolic complications, as well as increased risk of fatty liver disease and CVD. The present study investigated the underlying mechanism and doseresponse effects of resveratrol (RV) on obesity, hepatic steatosis and dyslipidaemia in mice fed a HFD. Male C57BL/6J mice were fed a normal diet or a HFD (20\% fat, w/w) combined with 0.005 or $0.02 \%(\mathrm{w} / \mathrm{w})$ RV for 10 weeks. As expected, mice fed a HFD developed obesity, as shown by increased body weight gain, visceral fat, hepatic fat and plasma cholesterol. RV significantly reduced visceral fat and plasma NEFA. In the liver of HFD-fed mice, RV significantly reduced TAG and cholesterol, as well as lipid droplet number and size. A low dose of RV (0.005\%) appeared to be more effective than a higher dose of RV (0.02\%) for suppressing adiposity and hepatic steatosis development with a significant decrease in body weight gain, plasma TAG and total cholesterol levels. These changes were seemingly attributable to a suppression of the fatty acid (FA) synthase, glucose-6-phosphate dehydrogenase, and phosphatidate phosphohydrolase and/or an activation of FA oxidation in the liver and epididymal adipose tissue. In conclusion, daily consumption of a low dose of $\mathrm{RV}$ is effective for protecting against diet-induced obesity, hepatic steatosis and dyslipidaemia in HFD-fed mice.
\end{abstract}

Key words: Resveratrol: High-fat diet: Adiposity: Hepatic steatosis: Lipid-regulating enzymes

Obesity is characterised by an increase in white adipose tissue (WAT) mass, which can result from an excess of food intake relative to energy expenditure. Obesity is also associated with hepatic steatosis and hyperlipidaemia, indicated by excessive accumulation of fat in the liver and excess fat in circulation $^{(1)}$. Central adiposity due to accumulation of visceral adipose tissue is linked to significantly increased risk of hepatic steatosis ${ }^{(2)}$. Increased NEFA flux from visceral fat to the liver is one of the suggested underlying mechanisms ${ }^{(3)}$. Most dietary approaches for obesity prevention or treatment attempt to limit consumption of a diet rich in saturated fat ${ }^{(4)}$. In contrast, an increase in fruit and vegetable intake can help decrease obesity, which may be attributable to their low-energy density, fibre content or phenolic compound enrichment $^{(5,6)}$.

Resveratrol (RV, 3,5, $4^{\prime}$-trihydroxystilbene) is one of the natural polyphenolic compounds mainly found in grape skin. $\mathrm{RV}$ is well known for its cardioprotective ${ }^{(7)}$, anti-cancer ${ }^{(8)}$, anti-inflammatory, antioxidant ${ }^{(9)}$ and phyto-oestrogenic ${ }^{(10)}$ properties. Some studies have suggested that RV may also have beneficial effects on obesity. For example, RV appears to inhibit the proliferation of pre-adipocytes in vitro ${ }^{(11)}$, and hence may suppress the production of new fat cells. In addition, RV appears to inhibit lipogenesis as well as lipid accumulation $^{(12)}$ in human liver cells and mature adipocytes ${ }^{(13,14)}$. While the evidence from cellular studies suggests that RV may suppress the production of new fat cells and prevent fat accumulation in liver cells and adipocytes, the effects of $\mathrm{RV}$ on obesity and steatosis in vivo have been far more equivocal $^{(15-20)}$.

Several in vivo studies reported that RV protected against diet-induced obesity ${ }^{(15,16)}$ and hepatic steatosis ${ }^{(17)}$ in murine models. For example, body weight gain and fat accumulation were suppressed in mice fed a high-fat diet (HFD) with high dietary RV intake $(400 \mathrm{mg} / \mathrm{kg}$ body weight per d) over 15 weeks. In these HFD-fed mice, RV up-regulated the silent

Abbreviations: FA, fatty acid; FAS, fatty acid synthase; G6PD, glucose-6-phosphate dehydrogenase; HFD, high-fat diet; ND, normal diet; PAP, phosphatidate phosphohydrolase; RV, resveratrol; SIRT1, silent information regulating 2 homologue 1; WAT, white adipose tissue.

*Corresponding author: M.-S. Choi, fax +82 53950 6229, email mschoi@knu.ac.kr

† These authors contributed equally to this work. 
information regulating 2 homologue 1 (SIRT1) gene, which is a key regulator of energy and metabolic homeostasis ${ }^{(15)}$. However, in other studies, RV was reported to have minimal or no effect on obesity and hepatic steatosis ${ }^{(18-20)}$. In obese Zucker rats, RV supplementation $(10 \mathrm{mg} / \mathrm{kg}$ body weight per d) over 8 weeks failed to reduce body weight, although $\mathrm{RV}$ reduced adipose tissue mass ${ }^{(18)}$. Similarly in mice, RV $(22.4 \mathrm{mg} / \mathrm{kg}$ body weight per d) supplementation for 15 months had no effect on body weight, although RV appeared to mimic the effects of energy restriction ${ }^{(20)}$. The lack of consensus on the in vivo effect of RV on diet-induced obesity and hepatic steatosis may have been due to varying doses, diet and duration in previous studies. Importantly, the potential mechanism underlying the anti-obesity and hepatoprotective effects of RV in vivo still remains unclear. In particular, few studies have determined the activities of enzymes involved in lipogenesis and fatty acid (FA) oxidation in the liver and adipose tissue of RV-supplemented, diet-induced obese mice.

Accordingly, we first determined whether RV (0.005 or $0.02 \%$ in diet) had any dose-dependent effects on body weight gain, visceral adiposity, hepatic steatosis and dyslipidaemia in HFD-fed mice, a well-established murine model of diet-induced obesity ${ }^{(21,22)}$. Secondly, we examined lipid metabolic enzyme activity in the liver and WAT to further elucidate potential underlying mechanisms responsible for the effects of RV on diet-induced obesity.

\section{Experimental methods}

\section{Animals and diets}

A total of forty male $\mathrm{C} 57 \mathrm{BL} / 6 \mathrm{~J}$ mice ( 4 weeks old) were purchased from the Jackson Laboratory. The animals were maintained in a room with controlled temperature $\left(20-23^{\circ} \mathrm{C}\right)$ and lighting (alternating $12 \mathrm{~h}$ periods of light and dark) and fed a pelletised commercial non-purified diet for 1 week after arrival. The mice were then randomly divided into four groups ( $n$ 10) and fed the respective experimental diets for 10 weeks, as shown in Table 1; normal diet (ND) control (ND, American Institute of Nutrition (AIN)-76 semisynthetic diet), HFD control (HFD, 20\% fat based on the AIN-76 diet plus $1 \%$ cholesterol, w/w), 0.02\% RV (HFD + high RV, 0.02\% RV with HFD, w/w, Sigma Chemical Company) and 0.005\% RV $($ HFD + low RV, w/w, 0.005\% RV with HFD, w/w). The HFD was formulated to provide $20 \%$ of the total energy from fat, by replacing carbohydrate energy with lard and maize oil, and had the same amount of vitamins and minerals per kJ as the ND. The mice had free access to food and distilled water during the experimental period. Food intake and body weight were measured daily. All animal procedures were approved by the Ethics Committee for animal studies at Kyungpook National University, Republic of Korea.

\section{Sampling}

At the end of the experimental period, the mice were anaesthetised with ketamine after a $12 \mathrm{~h}$ fast. Blood samples were collected in heparinised tubes from the inferior vena cava and stored at $-70^{\circ} \mathrm{C}$ before analysis of plasma biomarkers. The liver and visceral fat depots (epididymal, perirenal, mesentery and retroperitoneum) were removed, rinsed with a physiological saline solution, weighed and immediately stored at $-70^{\circ} \mathrm{C}$

\section{Lipid analyses}

Plasma NEFA were measured using a commercial assay kit (NEFA-Wako; Wako Pure Chemical Industries). Plasma TAG

Table 1. Composition of experimental diets (\% of diet, w/w)

\begin{tabular}{|c|c|c|c|c|}
\hline Ingredients & ND & $\mathrm{HFD}+0 \mathrm{RV}$ & HFD+ high RV & HFD+low RV \\
\hline Casein & 20 & 20 & 20 & 20 \\
\hline DL-Met & 0.3 & 0.3 & 0.3 & 0.3 \\
\hline Sucrose & 50 & 37 & 36.98 & 36.995 \\
\hline Cellulose & 5 & 5 & 5 & 5 \\
\hline AIN-mineral ${ }^{*}$ & 3.5 & $4 \cdot 2$ & $4 \cdot 2$ & 4.2 \\
\hline AIN-vitamin $†$ & 1 & 1.2 & $1 \cdot 2$ & $1 \cdot 2$ \\
\hline Choline bitartrate & 0.2 & 0.2 & 0.2 & 0.2 \\
\hline Maize starch & 15 & $11 \cdot 1$ & $11 \cdot 1$ & $11 \cdot 1$ \\
\hline Lard & & 17 & 17 & 17 \\
\hline Maize oil & 5 & 3 & 3 & 3 \\
\hline Cholesterol & & 1 & 1 & 1 \\
\hline RV & & & 0.02 & 0.005 \\
\hline Total (\%) & 100 & 100 & 100 & 100 \\
\hline Diet $(\mathrm{kJ} / 100 \mathrm{~g})$ & 1611.42 & 1893.52 & 1893.19 & 1893.44 \\
\hline Energy from fat (\%) & $11 \cdot 7$ & $39 \cdot 8$ & $39 \cdot 8$ & $39 \cdot 8$ \\
\hline
\end{tabular}

ND, normal diet; HFD, high-fat diet; RV, resveratrol; HFD+0 RV, HFD; HFD+high RV, HFD supplemented with $0.02 \%$ RV; HFD+low RV, HFD supplemented with $0.005 \%$ RV; AIN, American Institute of Nutrition.

*AIN-76 mineral mixture: calcium phosphate $500 \mathrm{~g} / \mathrm{kg}, \mathrm{NaCl} 74 \mathrm{~g} / \mathrm{kg}$, potassium citrate $2220 \mathrm{~g} / \mathrm{kg}$, potassium sulphate $52 \mathrm{~g} / \mathrm{kg}$ magnesium oxide $24 \mathrm{~g} / \mathrm{kg}$, manganous carbonate $3.5 \mathrm{~g} / \mathrm{kg}$, ferric citrate $6 \mathrm{~g} / \mathrm{kg}$, zinc carbonate $1.6 \mathrm{~g} / \mathrm{kg}$, cupric carbonate $0.3 \mathrm{~g} / \mathrm{kg}$, potassium iodate $0.01 \mathrm{~g} / \mathrm{kg}$, sodium selenite $0.01 \mathrm{~g} / \mathrm{kg}$, chromium potassium sulphate $0.55 \mathrm{~g} / \mathrm{kg}$, sucrose $118.03 \mathrm{~g} / \mathrm{kg}$.

† AIN-76 vitamin mixture: thiamin $\mathrm{HCl} 0.6 \mathrm{~g} / \mathrm{kg}$, riboflavin $0.6 \mathrm{~g} / \mathrm{kg}$, pyridoxine $\mathrm{HCl} 0.7 \mathrm{~g} / \mathrm{kg}$, niacin $3 \mathrm{~g} / \mathrm{kg}$, calcium pantothenate $1.6 \mathrm{~g} / \mathrm{kg}$, folic acid $0.2 \mathrm{~g} / \mathrm{kg}$, biotin $0.02 \mathrm{~g} / \mathrm{kg}$, vitamin $B_{12} 1 \mathrm{~g} / \mathrm{kg}$, vitamin A $(500000 \mathrm{IU} / \mathrm{g}) 0.8 \mathrm{~g} / \mathrm{kg}$, vitamin $D_{3}(400000 \mathrm{IU} / \mathrm{g})$ $0.25 \mathrm{~g} / \mathrm{kg}$, vitamin $\mathrm{E}$ acetate $(500 \mathrm{IU} / \mathrm{g}) 10 \mathrm{~g} / \mathrm{kg}$, menadione sodium bisulphite $0.08 \mathrm{~g} / \mathrm{kg}$, sucrose $981.15 \mathrm{~g} / \mathrm{kg}$. 
and total cholesterol were measured using commercial kits (Asan Pharmaceutical), based on the lipase-glycerol phosphate oxidase method ${ }^{(23)}$ and cholesterol oxidase method, respectively $^{(24)}$. Plasma apoB was also determined using a commercial kit (Nitto Boseki).

Hepatic lipids were extracted using the method of Folch et $a l .{ }^{(25)}$. The dried lipid residues were dissolved in $1 \mathrm{ml}$ of ethanol for cholesterol and TAG assays. Triton X-100 and sodium cholate solution were added to $200 \mu \mathrm{l}$ of the dissolved lipid solution to produce a final concentration of $3 \mathrm{~mm}$. The hepatic cholesterol and TAG levels were analysed with the same commercial kit as used in the plasma analysis (Asan Pharmaceutical).

\section{Lipid-regulating enzyme activities}

To measure lipid-regulating enzyme activities in the liver and epididymal WAT, enzyme sources were prepared according to the method reported by Hulcher \& Oleson ${ }^{(26)}$. FA synthase (FAS) activity was determined by a spectrophotometric assay according to the methods of Carl et $a l^{(27)}$; one unit of FAS activity represented the oxidation of $1 \mathrm{nmol}$ of $\mathrm{NADPH}$ per $\min$ at $30^{\circ} \mathrm{C}$. The glucose-6-phosphate dehydrogenase (G6PD) activity was determined using the method of Pitkänen et $a l^{(28)}$, based on the reduction of $1 \mathrm{nmol}$ NADP per min at $25^{\circ} \mathrm{C}$ measured at $340 \mathrm{~nm}$ using a spectrophotometer. Phosphatidate phosphohydrolase (PAP) activity was measured using the method of Walton \& Possmayer ${ }^{(29)}$ and PAP activity was expressed as $\mathrm{nmol} / \mathrm{min}$ per $\mathrm{mg}$ protein. FA $\beta$-oxidation was measured spectrophotometrically by monitoring the reduction of $\mathrm{NAD}$ to $\mathrm{NADH}$ in the presence of palmitoylCoA as described by Lazarow ${ }^{(30)}$. The amount of protein in enzyme sources was determined by the Bradford method ${ }^{(31)}$ using bovine serum albumin as the standard.

\section{Histopathological analysis}

Liver and epididymal WAT were removed from the mice and fixed in a buffer solution of $10 \%$ formalin. All fixed tissues were processed routinely for paraffin embedding and $4 \mu \mathrm{m}$ sections were prepared and stained with haematoxylin and eosin. Stained areas were viewed using an optical microscope (Zeiss Axioscope) with a magnifying power of $\times 200$ and epididymal adipocyte size was measured by using Motic Images Plus 2.0ML (Motic).

\section{Statistical analysis}

All data were presented as the mean and standard error. Statistical analysis was performed using software SPSS (version 11.0,
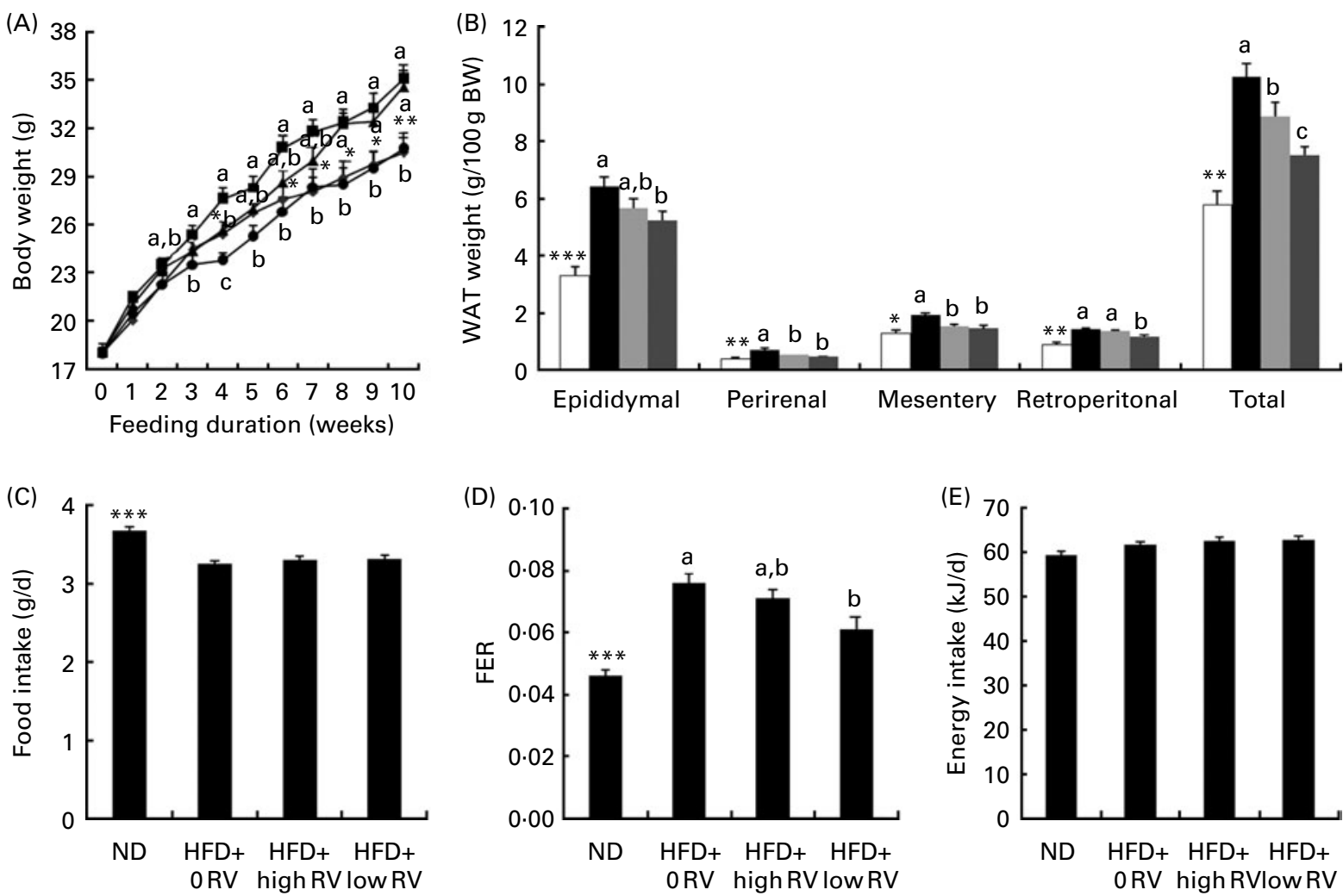

Fig. 1. Effects of resveratrol (RV) supplementation on (A) body weight $(\longrightarrow$, normal diet (ND); $\longrightarrow$, high-fat diet (HFD, HFD+0 RV); $\longrightarrow$, HFD supplemented with $0.02 \%$ RV (HFD+high RV); —— HFD supplemented with $0.005 \%$ RV (HFD+low RV)), (B) visceral white adipose tissue (WAT) ( $\square, \mathrm{ND}$; $\mathbf{\square}, \mathrm{HFD}+0 \mathrm{RV}$; $\square$, HFD+high RV; , HFD+low RV), (C) food intake weight, (D) food efficiency ratio (FER) and (E) energy intake in C57BL/6J mice fed a HFD. Values are means, with their standard errors represented by vertical bars. ${ }^{a, b, c}$ Mean values with unlike letters are significantly different among groups $(P<0.05)$. Mean values were significantly different for ND from those of HFD: ${ }^{\star} P<0.05,{ }^{\star \star} P<0.01,{ }^{\star \star \star} P<0.001$. Food efficiency ratio (FER) $=$ body weight gain/ food intake. 
SPSS, Inc.). Statistical differences between ND and HFD results were determined using Student's $t$ test. One-way ANOVA was performed to compare HFD groups with or without RV and Tukey's post hoc test was performed when significant differences were identified between the groups at $P<0 \cdot 05$.

\section{Results}

\section{Effects of resveratrol on body weight and food intake in diet-induced obese mice}

Food intake was significantly suppressed in mice fed a HFD alone compared to ND-fed mice, and daily energy intake was not different between the two groups (Fig. 1). However, body weight was significantly greater in the mice fed a HFD alone compared to ND-fed mice after 6 weeks. In HFD-fed mice, supplementation of RV at a low dose (0.005\%) significantly suppressed body weight gain after 3 weeks, but the higher dose of RV (0.02\%) was surprisingly not effective (Fig. 1). Neither food intake nor energy intake was affected by RV supplementation, whereas food efficiency ratio was significantly lower in the low-dose (0.005\%) RV group, compared to the HFD control group (Fig. 1).

(A)

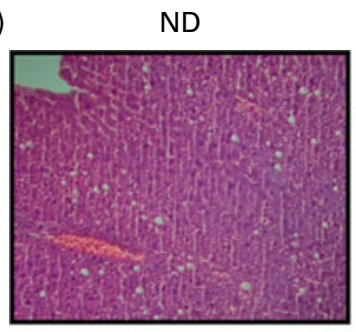

(B)

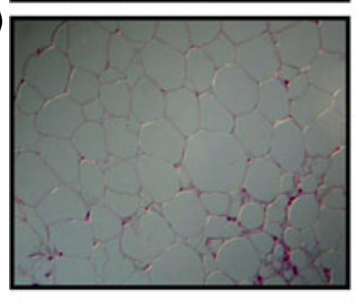

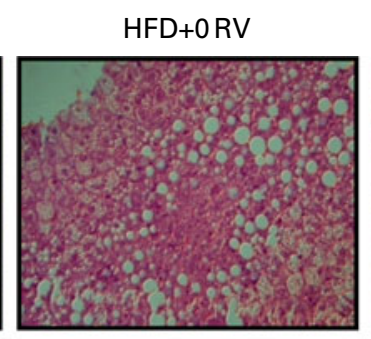

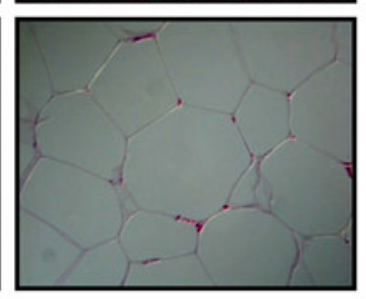

\section{Effects of resveratrol on fat accumulation in diet-induced obese mice}

HFD feeding resulted in a significant increase in total visceral WAT weight, including the epididymal, perirenal, retroperitoneal and mesentery depots compared to ND (Fig. 1). RV intake had differential effects on visceral adipose depots. Perirenal and mesentery adipose depots were significantly reduced in HFD-fed mice supplemented with either highdose or low-dose RV (Fig. 1). Only the low dose of RV (0.005\%) effectively reduced the weight of the epididymal and retroperitoneal adipose depots in HFD-fed mice (Fig. 1). Hence, overall total visceral adipose weight was significantly lowered by RV supplementation, although the low dose appeared to be more effective than the higher dose (Fig. 1). Morphological observations also indicated that epididymal adipocyte size was smaller in the RV-supplemented mice than in the HFD control mice (Fig. 2(B) and (C)).

\section{Effect of resveratrol on plasma and hepatic lipid levels in diet-induced obese mice}

Next, we determined if there was any evidence of a dosedependent effect of RV on plasma and hepatic lipid levels

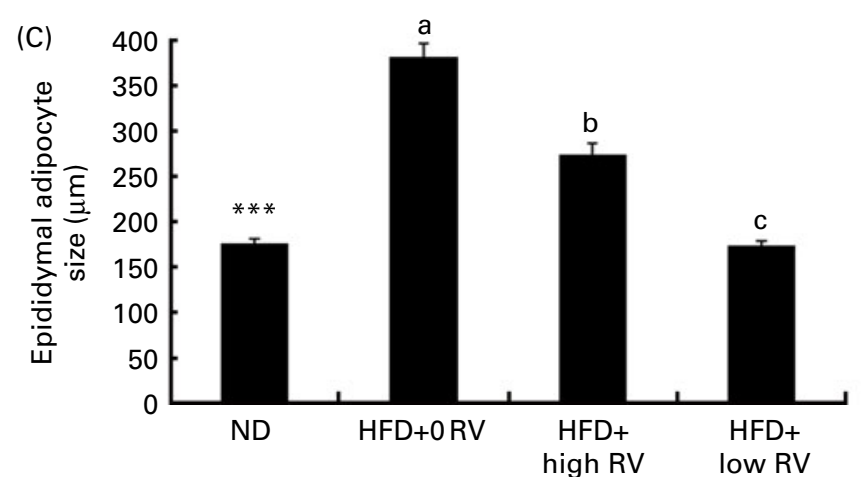

Fig. 2. Effects of resveratrol (RV) supplementation on (A) liver and (B) epididymal adipose tissue morphology in C57BL/6J mice fed a high-fat diet (HFD). Haematoxylin and eosin (H\&E)-stained transverse-section of the liver and epididymal fat; each liver and epididymal fat ( $n$ 10) were removed and wrapped with salinesoaked gauze after removing the connective tissues. All were fixed in $10 \%$ parafomaldehyde/PBS, embedded in paraffin, and then stained with H\&E. Original magnification $\times 200$. Effects of RV supplementation on epididymal adipocyte size in C57BL/6J mice fed a HFD. Values are means, with their standard errors represented by vertical bars. ${ }^{\text {a,b,c }}$ Mean values with unlike letters were significantly different $(P<0.05)$. ${ }^{* * *}$ Mean value was significantly different from those of the HFD groups $(P<0.001)$. ND, normal diet; HFD+0 RV, HFD; HFD+high RV, HFD supplemented with $0.02 \%$ RV; HFD+low RV, HFD supplemented with 0.005\% RV. (A colour version of this figure can be found online at http://www.journals.cambridge.org/bjn) 
Table 2. Effects of resveratrol (RV) supplementation on plasma and hepatic lipid levels in C57BL/6J mice fed a high-fat diet (HFD) (Mean values with their standard errors)

\begin{tabular}{|c|c|c|c|c|c|c|c|c|}
\hline & \multicolumn{2}{|c|}{ ND } & \multicolumn{2}{|c|}{$\mathrm{HFD}+0 \mathrm{RV}$} & \multicolumn{2}{|c|}{ HFD+high RV } & \multicolumn{2}{|c|}{ HFD+low RV } \\
\hline & Mean & SE & Mean & SE & Mean & SE & Mean & SE \\
\hline \multicolumn{9}{|c|}{ Plasma lipids (mmol/l) } \\
\hline NEFA & 1.00 & 0.04 & $1 \cdot 12^{\mathrm{a}}$ & 0.04 & $0.86^{\mathrm{b}}$ & 0.04 & $0.81^{\mathrm{b}}$ & 0.03 \\
\hline TAG & 0.97 & $0 \cdot 10$ & $1 \cdot 13^{a, b}$ & 0.07 & $1.37^{\mathrm{a}}$ & 0.14 & $0.94^{b}$ & 0.11 \\
\hline Total cholesterol & $3 \cdot 47^{\star \star \star}$ & 0.08 & $4 \cdot 81^{\mathrm{a}}$ & $0 \cdot 10$ & $4 \cdot 71^{a}$ & 0.18 & $4 \cdot 00^{\mathrm{b}}$ & 0.31 \\
\hline HDL-cholesterol & $0.83^{\star}$ & 0.01 & 1.16 & 0.08 & 1.16 & 0.06 & $1 \cdot 18$ & 0.03 \\
\hline Alt & $3 \cdot 13$ & 0.20 & $3 \cdot 18^{\mathrm{a}}$ & 0.01 & $3.03^{\mathrm{a}}$ & 0.01 & $2 \cdot 75^{\mathrm{b}}$ & 0.07 \\
\hline ApoB:apoA-I & $0 \cdot 13^{\star * *}$ & 0.001 & $0.21^{a}$ & 0.002 & $0 \cdot 19^{a, b}$ & 0.001 & $0 \cdot 13^{\mathrm{b}}$ & 0.001 \\
\hline \multicolumn{9}{|c|}{ Hepatic lipids $(\mu \mathrm{mol} / \mathrm{g})$} \\
\hline TAG & $10 \cdot 83^{\star \star \star}$ & 0.06 & $21 \cdot 89^{\mathrm{a}}$ & $1 \cdot 18$ & $18 \cdot 43^{b}$ & 0.93 & $14.93^{\mathrm{C}}$ & 0.44 \\
\hline Cholesterol & $2 \cdot 51^{\star \star \star}$ & 0.15 & $14 \cdot 64^{\mathrm{a}}$ & 0.58 & $7 \cdot 73^{b}$ & 0.64 & $5 \cdot 74^{\mathrm{c}}$ & $0 \cdot 10$ \\
\hline
\end{tabular}

ND, normal diet; HFD+0 RV, HFD; HFD+high RV, HFD supplemented with $0.02 \%$ RV; HFD+low RV, HFD supplemented with $0.005 \%$ RV; Al, atherogenic index.

a,b,c Mean values with unlike superscript letters are significantly different $(P<0.05)$.

Mean values were significantly different for ND from those of HFD: ${ }^{\star} P<0.05,{ }^{\star \star \star} P<0.001$.

$\dagger \mathrm{Al}=($ total cholesterol $-\mathrm{HDL}$-cholesterol)/HDL-cholesterol.

(Table 2). Plasma NEFA and TAG levels were not elevated in the mice fed the HFD alone compared to ND-fed mice. As a result of a concomitant increase in both plasma total cholesterol and HDL-cholesterol levels, atherogenic index was unchanged in the HFD control group compared to the ND group. However, the apoB:apoA-I ratio, which is a more sensitive biomarker to measure CHD than atherogenic index, was significantly higher in the HFD control group compared to the ND group. Despite the lack of changes in plasma lipid levels induced by the HFD, RV at both a low and high dose (0.005 and $0.02 \%$ ) significantly reduced plasma NEFA level compared to the HFD control group and the ND group. Remarkably, RV was more effective at a low dose (0.005\%), but not at a higher dose (0.02\%), for reducing plasma TAG and total cholesterol levels in HFD-fed mice. Plasma HDL-cholesterol level was not significantly different between mice fed the HFD alone and the two RV groups. However, the apoB:apoA-I ratio was significantly lower in the low-dose RV group compared to the HFD control group.

Hepatic TAG and cholesterol contents were increased approximately 2 -fold and 6-fold, respectively, in $\mathrm{C} 57 \mathrm{BL} / 6$ mice fed the HFD alone as previously observed by others ${ }^{(32,33)}$. In HFD-fed mice, RV significantly reduced hepatic TAG and cholesterol contents compared to the HFD control group, although not in a dose-dependent manner. Consistent with the observed hepatic TAG and cholesterol contents, haematoxylin and eosin staining of liver sections for lipids indicated that hepatic lipid accumulation was more pronounced in the HFD control mice compared to ND-fed mice (Fig. 2). RV caused a marked decrease in the number and size of liver fat droplets, and the low dose of RV (0.005\%) appeared to be more effective than the higher dose of RV (0.02\%).

\section{Effect of resveratrol on lipid-regulating enzyme activities in liver of diet-induced obese mice}

To further examine the mechanism via which RV suppresses lipid accumulation in the liver, we determined the activity of hepatic lipid-regulating enzymes. The activity of hepatic PAP, which is a rate-limiting enzyme in TAG synthesis, was elevated in mice fed the HFD alone compared to ND-fed mice. We observed no changes in de novo lipogenic FAS and G6PD activities in the liver of mice fed the HFD alone. However, FA oxidation was significantly augmented in the mice fed the HFD alone compared to ND-fed mice (Table 3). In contrast, the activity of PAP was significantly suppressed by RV in the liver of HFD-fed mice without affecting FA oxidation (Table 3). Furthermore, $0.02 \% \mathrm{RV}$ in the diet significantly decreased hepatic FAS activity by $43 \%$ compared to the HFD alone (Table 3 ).

\section{Effect of resveratrol on activities of enzymes involved in lipogenesis and fatty acid oxidation in epididymal white adipose tissue of diet-induced obese mice}

We next examined the activities of enzymes that can regulate lipid accumulation in epididymal WAT. C57BL/6J mice fed the HFD alone showed a significant increase of lipogenic enzyme PAP activity in epididymal WAT compared to NDfed mice, although no change in $\beta$-oxidation was observed in HFD-fed mice (Table 3). In contrast, RV significantly decreased FAS and PAP activities in epididymal WAT compared to the HFD-alone group (Table 3 ). Furthermore, mice fed a low dose of RV (0.005\%) showed a significant decrease in G6PD activity and a significant increase in FA $\beta$-oxidation in epididymal WAT compared to the mice fed the HFD alone (Table 3).

\section{Discussion}

A chronic HFD is a major contributing factor underlying the development of obesity in humans. Currently, there is widespread interest in natural compounds that can suppress dietinduced obesity. In the present study, we first determined whether RV exerted any dose-dependent effect on weight gain, hepatic steatosis or dyslipidaemia. Next, we determined whether RV affected lipid-regulating enzyme activity in the liver or adipose tissue. We used C57BL/6J mice which are prone to the development of obesity when fed a HFD, 
Table 3. Effects of resveratrol (RV) supplementation on lipid-regulating enzyme activities in the liver and adipose tissue of C57BL/6J mice fed a high-fat diet (HFD)

(Mean values with their standard errors)

\begin{tabular}{|c|c|c|c|c|c|c|c|c|}
\hline & \multicolumn{2}{|c|}{ ND } & \multicolumn{2}{|c|}{$\mathrm{HFD}+0 \mathrm{RV}$} & \multicolumn{2}{|c|}{ HFD+high RV } & \multicolumn{2}{|c|}{ HFD+low RV } \\
\hline & Mean & SE & Mean & SE & Mean & SE & Mean & SE \\
\hline \multicolumn{9}{|l|}{ Liver } \\
\hline FAS (nmol/min per mg protein) & 0.96 & 0.05 & $1 \cdot 22^{\mathrm{a}}$ & 0.12 & $0.92^{a, b}$ & $0 \cdot 11$ & $0.69^{b}$ & 0.01 \\
\hline G6PD (nmol/min per mg protein) & $4 \cdot 18$ & 0.67 & $3.46^{a}$ & 0.32 & $3.26^{a}$ & 0.08 & $1.71^{\mathrm{b}}$ & 0.18 \\
\hline PAP (nmol/min per mg protein) & $17 \cdot 62^{*}$ & 0.54 & $22 \cdot 98^{\mathrm{a}}$ & 1.39 & $18.56^{\mathrm{b}}$ & 0.26 & $17 \cdot 37^{b}$ & $0 \cdot 12$ \\
\hline$\beta$-Oxidation ( $\mathrm{nmol} / \mathrm{min}$ per $\mathrm{mg}$ protein) & $1.56^{\star}$ & 0.07 & 2.43 & 0.28 & 3.03 & 0.02 & $3 \cdot 13$ & 0.43 \\
\hline \multicolumn{9}{|l|}{ Adipose tissue } \\
\hline FAS (nmol/min per mg protein) & $42 \cdot 50$ & $3 \cdot 21$ & $37 \cdot 17^{\mathrm{a}}$ & 1.77 & $23 \cdot 65^{\mathrm{b}}$ & 1.42 & $15 \cdot 00^{\mathrm{c}}$ & 1.29 \\
\hline G6PD (nmol/min per mg protein) & $27 \cdot 10$ & 1.78 & $32 \cdot 33^{a}$ & 1.66 & $26 \cdot 59^{\mathrm{a}}$ & 1.68 & $20 \cdot 35^{\mathrm{b}}$ & 0.58 \\
\hline PAP (nmol/min per mg protein) & $251 \cdot 64^{\star *}$ & 50.49 & $351 \cdot 96^{\mathrm{a}}$ & 16.99 & $248 \cdot 27^{b}$ & 7.95 & $195 \cdot 29^{\mathrm{c}}$ & $15 \cdot 25$ \\
\hline$\beta$-Oxidation ( $\mathrm{nmol} / \mathrm{min}$ per $\mathrm{mg}$ protein) & 123.37 & 63.30 & $141 \cdot 93^{\mathrm{a}}$ & $10 \cdot 34$ & $98 \cdot 95^{\mathrm{a}}$ & $31 \cdot 10$ & $364 \cdot 27^{\mathrm{b}}$ & $57 \cdot 80$ \\
\hline
\end{tabular}

ND, normal diet; HFD+0 RV, HFD; HFD+high RV, HFD supplemented with $0.02 \%$ RV; HFD+low RV, HFD supplemented with $0.005 \%$ RV; FAS, fatty acid synthase; G6PD, glucose-6-phosphate dehydrogenase; PAP, phosphatidate phosphohydrolase.

a,b,c Mean values with unlike superscript letters are significantly different $(P<0.05)$.

Mean values were significantly different for ND from those of HFD: ${ }^{\star} P<0.05$, ${ }^{\star \star} P<0.01$.

developing similar characteristics as human obesity, compared to genetically obese animals ${ }^{(21,22,34)}$. We found that RV protected against diet-induced obesity, but observed unexpectedly no dose-response effects.

As others have shown ${ }^{(21,22,34)}$, the HFD resulted in a marked increase of body weight gain due to increased adiposity, despite significantly decreased food intake. RV significantly suppressed adiposity in HFD-fed mice, due to lower visceral WAT weight. Consistent with these results, we observed that epididymal WAT size was smaller in HFD-fed mice supplemented with RV. RV supplemented at a lower dose was more effective than the higher dose at suppressing visceral fat accumulation. Furthermore, RV at a low dose, but not a high dose, significantly suppressed body weight gain compared to HFD control mice. Past animal studies have been equivocal, with some studies reporting that RV suppresses body weight gain and fat accumulation in HFD-fed mice $^{(15,16)}$ and rats ${ }^{(17)}$, while several studies have reported that RV has no effect on body weight gain ${ }^{(18-20)}$. The discrepancies between past studies may be attributable to differences in diet composition, duration of diet, dosage and experimental animals used. Few past studies have examined any dose-response effects of RV in HFD-fed animals. One previous study in rats fed a hyperenergetic diet containing different doses $(6,30,60 \mathrm{mg} / \mathrm{kg}$ body weight per d) of $\mathrm{RV}$ for 6 weeks reported no dose-response effects on adipose weight $^{(35)}$. Importantly, our study compared two doses of $\mathrm{RV}$, both below $0.02 \%$, equivalent to $2 \mathrm{mg} / \mathrm{kg}$ body weight per $d$. Our findings suggest that RV may be an effective antiobesity agent, reducing body weight and fat accumulation in response to a HFD, and that $0.005 \% \mathrm{RV}$ may be an adequate amount for suppressing body weight gain and adiposity, although a response study with lower doses of RV is further needed to determine the minimum effective dose.

Next, we examined potential mechanisms underlying the body weight and adiposity-suppressing effect of RV. RV did not lead to decreased food intake, because no significant difference in food intake was observed in the HFD-fed mice regardless of RV administration. Many studies have reported that dietary fat led to augmentation of lipogenic enzyme $\operatorname{activity}^{(36,37)}$, although contradictory findings have been reported $^{(38)}$. WAT is a major site of de novo FA synthesis, and the FA synthesised in WAT can be either re-esterified to TAG within WAT or oxidised via mitochondrial $\beta$-oxidation along with FA from the diet. FAS plays a central role in de novo lipogenesis by catalysing the synthesis of saturated long-chain FA from acetyl-CoA, malonyl-CoA and NADPH. G6PD is also a lipogenic enzyme involved in supplying NADPH for the biosynthesis of FA. In the present study, no changes in FAS or G6PD activity as well as FA $\beta$-oxidation activity were observed in epididymal WAT of ND-fed mice compared to mice fed a HFD alone. However, we observed a significant increase in PAP activity, a rate-limiting enzyme in TAG synthesis in epididymal WAT. These findings indicate that HFD does not promote de novo FA synthesis and fat oxidation in adipose tissue. The increased epididymal WAT mass in HFD-fed mice may be attributable to increased esterification of NEFA provided by the diet. Interestingly, in HFD-fed mice, RV administration significantly inhibited the activity of de novo lipogenic enzymes including FAS and G6PD as well as PAP in epididymal WAT. Again we observed no dose-response effect, and 0.005\% RV significantly augmented FA $\beta$-oxidation in epididymal WAT, which may help explain the lower visceral adiposity compared to $0.02 \%$ RV-supplemented mice. Our findings are consistent with a previous study that reported suppression of FAS gene expression and protein level by RV in epididymal WAT of HFD-fed mice ${ }^{(39)}$. Furthermore, RV has been reported to inhibit de novo lipogenesis in parallel with the down-regulation of lipogenic gene expression in human adipocytes ${ }^{(13)}$. Taken together, the current evidence indicates that the suppression of adiposity by RV may be due to the inhibition of de novo FA synthesis or stimulation of FA oxidation in WAT.

Another possible mechanism by which dietary RV ameliorates adiposity may be due to the activation of lipolysis. Lipolysis is one of the important metabolic pathways regulating TAG accumulation in WAT and two major enzymes, hormone-sensitive lipase and adipose TAG lipase, are involved 
in adipose TAG hydrolysis. Rayalam et $a l .{ }^{(40)}$ have shown that RV down-regulated the expression of hormone-sensitive lipase as well as lipogenic gene in WAT, indicating that RV may alter fat mass by directly affecting the biochemical pathways involved in adipogenesis. In contrast, a recent study reported that RV induced the release of NEFA but not glycerol in 3T3 L1 cells and up-regulated adipose TAG lipase mRNA and protein expression without affecting hormone-sensitive lipase ${ }^{(41)}$. Furthermore, NEFA release was increased by RV in epididymal WAT of wild-type and hormone-sensitive lipase knockout mice, but no changes were observed in epididymal WAT of adipose TAG lipase knockout mice, suggesting that adipose TAG lipase seems to be the main target for the lipolytic effect of $\mathrm{RV}^{(41)}$

Fat accumulation in adipose tissue is closely associated with the development of hepatic steatosis, which is an excessive accumulation of fat in the liver. Several mechanisms may lead to a hepatic steatosis: (1) increased NEFA supply due to increased lipolysis from WAT and/or increased intake of dietary fat, (2) increased de novo hepatic lipogenesis, (3) decreased fatty oxidation in the liver and (4) decreased hepatic VLDL-TAG secretion ${ }^{(42)}$. Excessive consumption of dietary fat, especially SFA, leads to an excess accumulation of TAG in the liver ${ }^{(32,33)}$. We also observed that the HFD led to a significant increase of hepatic TAG content as well as hepatic PAP activity, consistent with a previous observation that SFA dramatically increased $P A P$ gene expression ${ }^{(43)}$. However, the HFD did not lead to increased de novo lipogenic FAS and G6PD activities in the liver, or lead to increased plasma
NEFA and TAG levels compared to ND-fed mice. In agreement with the present study, plasma NEFA and TAG levels, as well as hepatic FAS activity and FAS gene expression, were not increased in HFD (38.1\% fat, w/w) -fed C57BL/6 mice after 18 weeks ${ }^{(44)}$. Furthermore, Kim et al. ${ }^{(45)}$ reported that a HFD did not affect plasma NEFA and TAG levels in C57BL/6 mice, although the HFD induced hepatic steatosis and adiposity. Moreover, Kim et al. ${ }^{(45)}$ showed that genes related to FA $\beta$-oxidation were activated in the liver of HFD-fed mice, consistent with our data. Accordingly, these findings suggest that a HFD can promote hepatic lipogenesis by esterification of NEFA provided from the diet but not from de novo lipogenesis in the liver. Hepatic TAG accumulation may lead to augmentation of FA oxidation by a feedback mechanism. We found that RV significantly reduced hepatic steatosis and TAG content as well as plasma NEFA level in HFD-fed mice. The suppression of hepatic lipogenesis by RV was linked to decreased activities of hepatic de novo lipogenic FAS as well as PAP without affecting FA oxidation. The low dose of RV appeared to be more effective for suppressing hepatic steatosis, similar to body weight and fat. Taken together, these findings suggest that the beneficial effect of RV on hepatic steatosis is mediated, at least in part, by a decrease in de novo FA synthesis and esterification based on the evidence of lower hepatic FAS and PAP activity. Previous studies also indicate that RV may prevent hepatic steatosis by regulating hepatic lipid metabolism-related genes such as FAS and SIRT1 ${ }^{(46)}$. Also, the lowered plasma NEFA may account in part for the decrease in hepatic TAG content in mice supplemented with RV, since

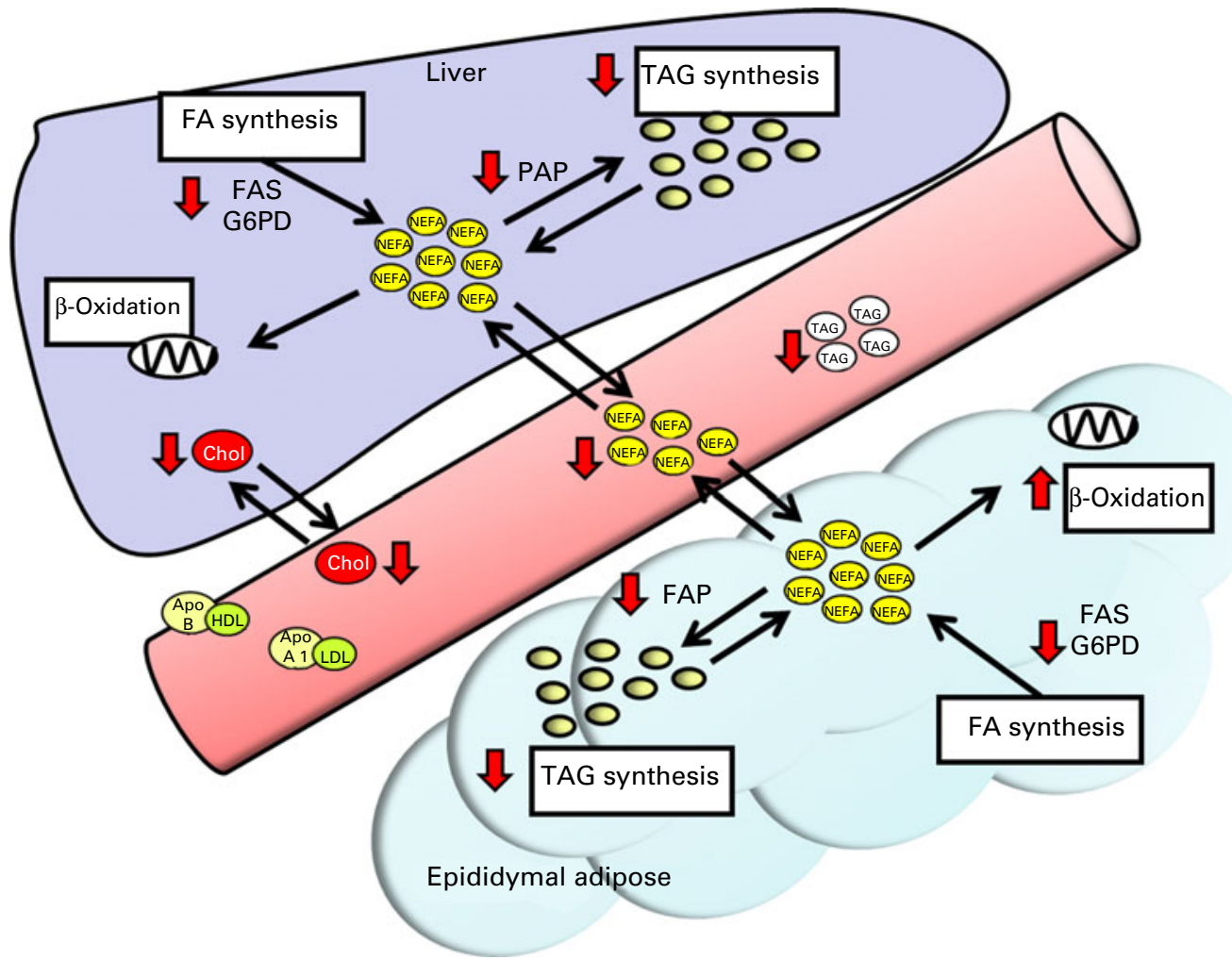

Fig. 3. Summary of the effects of resveratrol supplementation on lipid metabolism in the liver and adipose tissue. $\Omega$, Change in activity or concentration. FA, fatty acid; FAS, fatty acid synthase; G6PD, glucose-6-phosphate dehydrogenase; PAP, phosphatidate phosphohydrolase; Chol, cholesterol. (A colour version of this figure can be found online at http://www.journals.cambridge.org/bjn) 
hepatic TAG accumulation during insulin resistance occurs as a result of the increase in the supply of circulating NEFA to the liver as well as through an increased endogenous lipogenesis ${ }^{(42)}$.

In the present study, we observed that the HFD resulted in an increased plasma apoB:apoA-I ratio, as well as total cholesterol concentration mostly due to increased HDL-cholesterol concentration. The increase in total cholesterol and HDLcholesterol is a common feature of most mouse strains fed a $\mathrm{HFD}^{(47)}$. Plasma apoB or apoA-I concentrations independently or together as a ratio (apoB:apoA-I) are reported to predict cardiovascular risk more accurately than lipid levels ${ }^{(48)}$. Previously we reported that long-term RV supplementation over 20 weeks significantly decreased plasma total cholesterol and LDL-cholesterol levels in apoE knockout mice fed an ND, whereas the apoB:apoA-I ratio was increased ${ }^{(49)}$. Herein, we observed that $0.005 \%$ RV normalised the HFD-mediated increase of plasma total cholesterol and apoB:apoA-I ratio, whereas the beneficial effects were not observed in HFD-fed mice supplemented with $0.02 \% \mathrm{RV}$. Zern et al. ${ }^{(50)}$ reported that the grape polyphenol failed to improve plasma total cholesterol level, although hepatic cholesterol metabolism was altered based on evidence of lower hepatic acyl-CoA: cholesterol acyltransferase (ACAT) activity, a key cholesterolregulating enzyme involved in the esterification and absorption of cholesterol ${ }^{(51)}$. Furthermore, grape polyphenol was suggested to decrease the secretion of hepatic LDL-cholesterol reducing cholesterol accumulation in the arterial wall ${ }^{(51)}$. Consistent with this study, we found that both lower and higher RV significantly lowered hepatic cholesterol content by 47 and $61 \%$, respectively, as compared to the HFD-alone group. Although we did not determine hepatic 3-hydroxy-3methylglutaryl (HMG)-CoA reductase activity and faecal bile acid levels in this study, the inhibition of hepatic HMG-CoA reductase activity or the increase of faecal bile acids excretion may be related to the plasma and hepatic cholesterol-lowering effect of RV. In our previous study, we demonstrated that RV led to decreased cholesterol level in plasma as well as the liver by suppressing the activity of hepatic HMG-CoA reductase, a rate-limiting enzyme for cholesterol synthesis, in apoE-deficient mice ${ }^{(49)}$. On the other hand, Zhu et al. ${ }^{(52)}$ reported that RV significantly lowered cholesterol levels in the serum and liver of HFD-fed rats and these effects resulted from the enhanced excretion of bile acids into faeces. Another possibility is an inhibition of pancreatic bile salt-dependent lipase by $\mathrm{RV}^{(53)}$. In rat pancreatic $A R 4-2 J$ cells, $R V$ inhibited the secretion and activity of bile salt-dependent lipase which is synthesised in pancreatic cells and involved in duodenal hydrolysis of lipid esters and absorption of non-esterified cholesterol.

Overall, our findings suggest that lower doses of RV may have more beneficial effects on adiposity, hepatic steatosis and hyperlipidaemia in HFD-fed mice compared to higher doses of RV. Currently, it is unclear why RV has a reverse dose-response effect, but it may explain why previous studies on the in vivo effects of RV have been equivocal. One possibility suggested in a recent review is that RV at high doses can exhibit pro-oxidant properties ${ }^{(54)}$. Oxidative stress closely correlates with fat accumulation in humans and mice ${ }^{(55)}$ and reactive oxygen species lead to mitochondrial dysfunction associated with obesity and obesity-related metabolic disease $^{(56)}$. Considerable evidence supports that high doses of polyphenols can potentially cause adverse metabolic effects through pro-oxidative effects ${ }^{(57)}$. In fact, several recent papers indicate that high doses of RV in vivo can lead to adverse physiological effects. Dudley et al. ${ }^{(58)}$ reported that at lower doses $(2.5$ or $5 \mathrm{mg} / \mathrm{kg}$ body weight per $\mathrm{d}$ ), RV protected against heart ischaemia by inducing a survival signal causing the up-regulation of anti-apoptotic and redox proteins, while at higher doses ( $>25 \mathrm{mg} / \mathrm{kg}$ body weight per d) RV potentiated a death signal causing the down-regulation of redox proteins and up-regulation of pro-apoptotic proteins. Consistently, RV protected the heart when administered at relatively low doses between 2.5 and $10 \mathrm{mg} / \mathrm{kg}$ body weight ${ }^{(59)}$. In addition, high doses of trans-RV in hypercholesterolaemic rabbits have been reported to cause atherosclerotic lesions while lower doses of RV were found to be protective ${ }^{(60)}$.

In conclusion, the present findings suggest that dietary RV may be an effective anti-obesity agent and also protect against atherosclerosis when supplemented at low doses. RV decreases adiposity and hepatic steatosis in diet-induced obese mice and these effects appear to be mediated via regulation of lipid metabolism-related enzyme activity in the liver and adipose tissue, as summarised in Fig. 3. Low-dose RV was more beneficial for suppressing visceral adiposity and hepatic steatosis, along with protecting against dyslipidaemia in HFD-fed mice. In future studies, it will be important to establish whether varying doses of RV in humans consuming a HFD can provide any protection against hepatic steatosis, dyslipidaemia and visceral adipose tissue accumulation.

\section{Acknowledgements}

The present study was supported by the National Research Foundation of Korea grant funded by the Korea government (no. 531-2006-1-C00064 and no. 2011-0000912). None of the authors had any conflict of interest. S.-J. C. carried out the experiments and U. J. J. wrote the original manuscript with S.-J. C. M.-S. C. performed substantial editing as well as the design of the experiment. All authors reviewed the literature and contributed to the final report.

\section{References}

1. Haslam DW \& James WPT (2005) Obesity. Lancet 366, 1197-1209.

2. Thomas EL, Hamilton G, Patel N, et al. (2005) Hepatic triglyceride content and its relation to body adiposity: a magnetic resonance imaging and proton magnetic resonance spectroscopy study. Gut 54, 122-127.

3. Kuriyama H, Yamashita S, Shimomura I, et al. (1998) Enhanced expression of hepatic acyl-coenzyme A synthetase and microsomal triglyceride transfer protein messenger RNAs in the obese and hypertriglyceridemic rat with visceral fat accumulation. Hepatology 27, 557-562.

4. Walker CG, Zariwala MG, Holness MJ, et al. (2007) Diet, obesity and diabetes: a current update. Clin Sci 112, 93-111. 
5. Astrup A, Dyerberg J, Selleck M, et al. (2008) Nutrition transition and its relationship to the development of obesity and related chronic diseases. Obes Rev 1, 48-52.

6. Rastmanesh R (2011) High polyphenol, low probiotic diet for weight loss because of intestinal microbiota interaction. Chem Biol Interact 189, 1-8.

7. Hung LM, Chen JK, Huang SS, et al. (2000) Cardioprotective effect of resveratrol, a natural antioxidant derived from grapes. Cardiovasc Res 47, 549-555.

8. El-Mowafy AM \& Alkhalaf M (2003) Resveratrol activates adenylyl-cyclase in human breast cancer cells: a novel, estrogen receptor-independent cytostatic mechanism. Carcinogenesis 24, 869-873.

9. de la Lastra CA \& Villegas I (2007) Resveratrol as an antioxidant and pro-oxidant agent: mechanisms and clinical implications. Biochem Soc Trans 35, 1156-1160.

10. Bowers JL, Tyulmenkov VV, Jernigan SC, et al. (2000) Resveratrol acts as a mixed agonist/antagonist for estrogen receptors alpha and beta. Endocrinology 141, 3657-3667.

11. Hsu CL \& Yen GC (2006) Induction of cell apoptosis in 3T3-L1 pre-adipocytes by flavonoids is associated with their antioxidant activity. Mol Nutr Food Res 50, 1072-1079.

12. Shang J, Chen LL, Xiao FX, et al. (2008) Resveratrol improves non-alcoholic fatty liver disease by activating AMP-activated protein kinase. Acta Pharmacol Sin 29, 698-706.

13. Fischer-Posovszky P, Kukulus V, Tews D, et al. (2010) Resveratrol regulates human adipocyte number and function in a Sirt1-dependent manner. Am J Clin Nutr 92, 5-15.

14. Szkudelska K, Nogowski L \& Szkudelski T (2009) Resveratrol, a naturally occurring diphenolic compound, affects lipogenesis, lipolysis and the antilipolytic action of insulin in isolated rat adipocytes. J Steroid Biochem Mol Biol 113, $17-24$.

15. Lagouge M, Argmann C, Grhart-Hines Z, et al. (2006) Resveratrol improves mitochondrial function and protects against metabolic disease by activating SIRT1 and PGC-1 $\alpha$. Cell 127, $1-14$

16. Kim S, Jin Y, Choi Y, et al. (2011) Resveratrol exerts antiobesity effects via mechanisms involving down-regulation of adipogenic and inflammatory processes in mice. Biochem Pharmacol 81, 1343-1351.

17. Shang J, Chen LL \& Xiao FX (2008) Resveratrol improves high-fat induced nonalcoholic fatty liver in rats. Zhonghua Gan Zang Bing Za Zhi 16, 616-619.

18. Rivera L, Morón R, Zarzuelo A, et al. (2009) Long-term resveratrol administration reduces metabolic disturbances and lowers blood pressure in obese Zucker rats. Biochem Pharmacol 77, 1053-1106.

19. Tauriainen E, Luostarinen M, Martonen E, et al. (2011) Distinct effects of calorie restriction and resveratrol on diet-induced obesity and fatty liver formation. $J$ Nutr Metab 525094 (epublication 1 October 2011).

20. Baur JA, Pearson KJ, Price NL, et al. (2006) Resveratrol improves health and survival of mice on a high-calorie diet. Nature 444, 337-342.

21. West DB, Boozer CN, Moody DL, et al. (1992) Dietary obesity in nine inbred mouse strains. Am J Physiol 262, R1025-R1032.

22. Ghibaudi L, Cook J, Farley C, et al. (2002) Fat intake affects adiposity, comorbidity factors, and energy metabolism of Sprague-Dawley rats. Obes Res 10, 956-963.

23. McGowan MW, Artiss JD, Strandbergh DR, et al. (1983) A peroxidase-coupled method for the colorimetric determination of serum triglycerides. Clin Chem 29, 538-542.

24. Allain CC, Poon LS, Chan CS, et al. (1974) Enzymatic determination of total serum cholesterol. Clin Chem 20, 470-475.
25. Folch J, Lees M \& Sloane Stanley GH (1957) A simple method for the isolation and purification of total lipides from animal tissues. J Biol Chem 226, 497-509.

26. Hulcher FH \& Oleson WH (1973) Simplified spectrophotometric assay for microsomal 3-hydroxy-3-methylglutaryl CoA reductase by measurement of coenzyme A. J Lipid Res 14, 625-631.

27. Carl MN, Lakshmanan MR \& Porter JW (1975) FAS from rat liver. Methods Enzymol 35, 37-44.

28. Pitkänen E, Pitkänen O \& Uotila L (1997) Enzymatic determination of unbound D-mannose in serum. Eur J Clin Chem Clin Biochem 35, 761-766.

29. Walton PA \& Possmayer F (1985) Mg2-dependent phosphatidate phosphohydrolase of rat lung: development of an assay employing a defined chemical substrate which reflects the phosphohydrolase activity measured using membranebound substrate. Anal Biochem 151, 479-486.

30. Lazarow PB (1981) Assay of peroxisomal beta-oxidation of fatty acids. Methods Enzymol 72, 315-319.

31. Bradford MM (1976) A rapid and sensitive method for the quantitation of microgram quantities of protein utilizing the principle of protein-dye binding. Anal Biochem 72, 248-254.

32. Wierzbicki M, Chabowski A, Zendzian-Piotrowska M, et al. (2009) Chronic, in vivo, PPARalpha activation prevents lipid overload in rat liver induced by high fat feeding. $A d v$ Med Sci 54, 59-65.

33. Oosterveer MH, van Dijk TH, Tietge UJ, et al. (2009) High fat feeding induces hepatic fatty acid elongation in mice. PLoS One 4, e6066.

34. Collins S, Martin TL, Surwit RS, et al. (2004) Genetic vulnerability to diet-induced obesity in the $\mathrm{C} 57 \mathrm{BL} / 6 \mathrm{~J}$ mouse: physiological and molecular characteristics. Physiol Behav 81, 243-248.

35. Macarulla MT, Alberdi G, Gómez S, et al. (2009) Effects of different doses of resveratrol on body fat and serum parameters in rats fed a hypercaloric diet. $J$ Physiol Biochem 65, 369-376.

36. Nelson GJ, Kelley DS, Schmidt PC, et al. (1987) The influence of dietary fat on the lipogenic activity and fatty acid composition of rat white adipose tissue. Lipids 22, 338-344.

37. Rodríguez VM, Macarulla MT, Chávarri M, et al. (2001) Lipoprotein lipase and lipogenic enzyme activities in adipose tissue from rats fed different lipid sources. J Physiol Biochem 57, 245-254.

38. Jiang L, Wang Q, Yu Y, et al. (2009) Leptin contributes to the adaptive responses of mice to high-fat diet intake through suppressing the lipogenic pathway. PLoS One 4, e6884.

39. Kim S, Jin Y, Choi Y, et al. (2011) Resveratrol exerts antiobesity effects via mechanisms involving down-regulation of adipogenic and inflammatory processes in mice. Biochem Pharmacol 81, 1343-1351.

40. Rayalam S, Yang JY, Ambati S, et al. (2008) Resveratrol induces apoptosis and inhibits adipogenesis in 3T3-L1 adipocytes. Phytother Res 22, 1367-1371.

41. Lasa A, Schweiger M, Kotzbeck P, et al. (2011) Resveratrol regulates lipolysis via adipose triglyceride lipase. $J$ Nutr Biochem (epublication ahead of print version 2 May 2011).

42. Duval C, Muller M \& Kersten S (2007) PPARR and dyslipidemia. Biochim Biophys Acta 1771, 961-971.

43. Martin PG, Guillou H, Lasserre F, et al. (2007) Novel aspects of PPARalpha-mediated regulation of lipid and xenobiotic metabolism revealed through a nutrigenomic study. Hepatology 45, 767-777.

44. Sato A, Kawano H, Notsu T, et al. (2010) Antiobesity effect of eicosapentaenoic acid in high-fat/high-sucrose diet-induced obesity: importance of hepatic lipogenesis. Diabetes $\mathbf{5 9}$, 2495-2504. 
45. Kim S, Sohn I, Ahn JI, et al. (2004) Hepatic gene expression profiles in a long-term high-fat diet-induced obesity mouse model. Gene 340, 99-109.

46. Ajmo JM, Liang X, Rogers CQ, et al. (2008) Resveratrol alleviates alcoholic fatty liver in mice. Am J Physiol Gastrointest Liver Physiol 295, G833-G842.

47. Paigen B (1995) Genetics of responsiveness to high-fat and high-cholesterol diets in the mouse. Am J Clin Nutr 62, 458-62S.

48. Davidson MH (2009) Apolipoprotein measurements: is more widespread use clinically indicated? Clin Cardiol 32, 482-486.

49. Do GM, Kwon EY, Kim HJ, et al. (2008) Long-term effects of resveratrol supplementation on suppression of atherogenic lesion formation and cholesterol synthesis in apo E-deficient mice. Biochem Biophys Res Commun 374, 55-59.

50. Zern TL, West KL \& Fernandez ML (2003) Grape polyphenols decrease plasma triglycerides and cholesterol accumulation in the aorta of ovariectomized guinea pigs. J Nutr 133, $2268-2272$

51. Suckling KE \& Stange EF (1985) Role of acyl-CoA, cholesterol acyl-transferase in cellular cholesterol metabolism. J Lipid Res 26, 647-671.

52. Zhu L, Luo X \& Zin Z (2008) Effect of resveratrol on serum and liver lipid profile and antioxidant activity in hyperlipidemia rats. Asian-Aust J Anim Sci 21, 890-895.
53. Sbarra V, Ristorcelli E, Petit-Thévenin JL, et al. (2005) In vitro polyphenol effects on activity, expression and secretion of pancreatic bile salt-dependent lipase. Biochim Biophys Acta 1736, 67-76

54. de la Lastra CA \& Villegas I (2007) Resveratrol as an antioxidant and pro-oxidant agent: mechanisms and clinical implications. Biochem Soc Trans 35, 1156-1160.

55. Furukawa S, Fujita T, Shimabukuro M, et al. (2004) Increased oxidative stress in obesity and its impact on metabolic syndrome. J Clin Invest 114, 1752-1761.

56. Qatanani M \& Lazar MA (2007) Mechanisms of obesityassociated insulin resistance: many choices on the menu. Genes Dev 21, 1443-1455.

57. Martin KR \& Appel CL (2010) Polyphenols as dietary supplements: a double-edged sword. Nutr Diet Suppl 2, 1-12.

58. Dudley J, Das S, Mukherjee S, et al. (2009) Resveratrol, a unique phytoalexin present in red wine, delivers either survival signal or death signal to the ischemic myocardium depending on dose. J Nutr Biochem 20, 443-452.

59. Das S, Fraga CG \& Das DK (2006) Cardioprotective effect of resveratrol via HO-1 expression involves p38 map kinase and PI-3-kinase signaling, but does not involve NF kappaB. Free Radic Res 40, 1066-1075.

60. Wilson T, Knight TJ, Beitz DC, et al. (1996) Resveratrol promotes atherosclerosis in hypercholesterolemic rabbits. Life Sci 59, 15-21. 